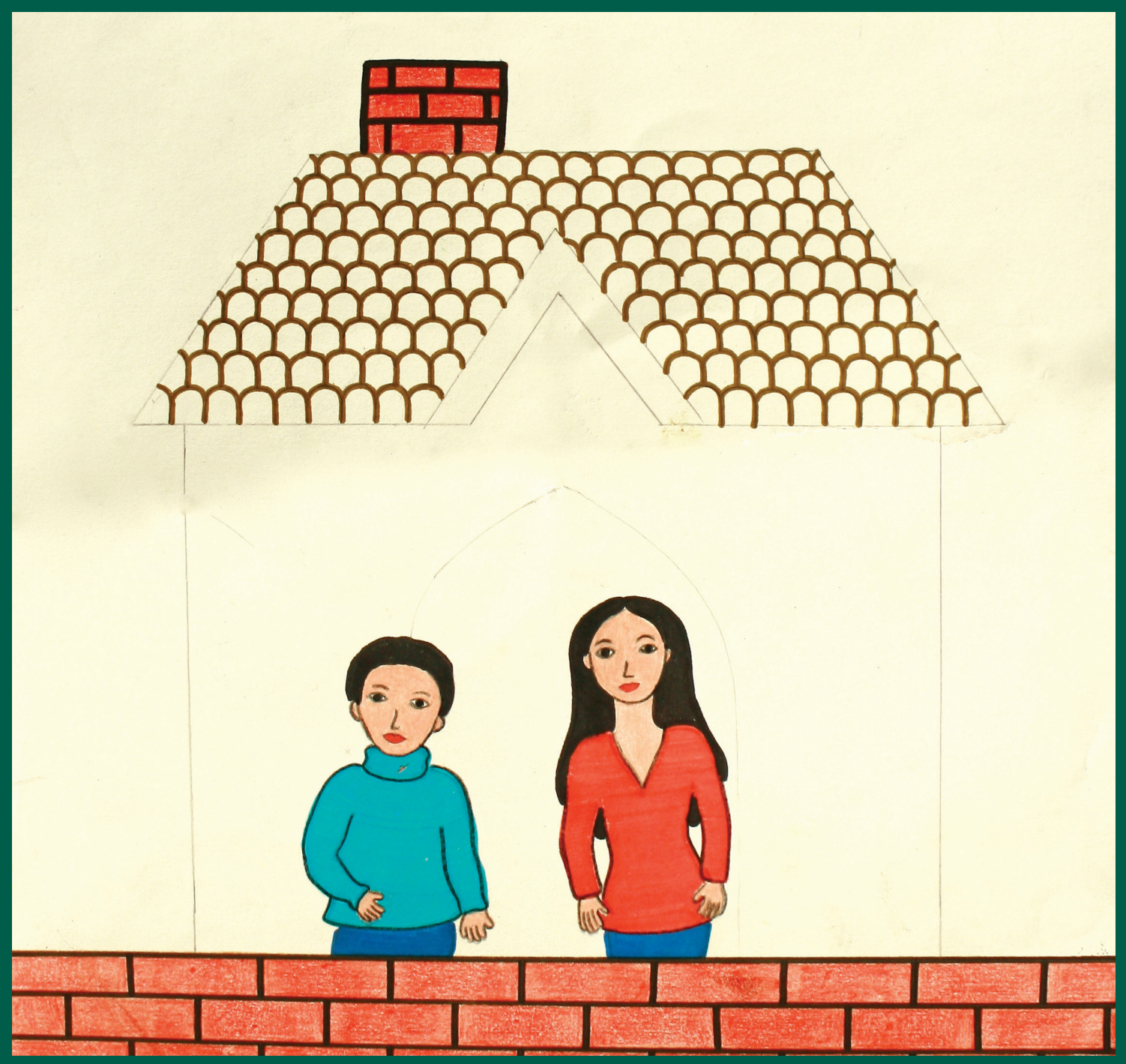

Ariatna Barrios - Arte sin Fronteras - Programa Talentos Especiales

Lisbeth Liliana Dottor Dotor

Fonoaudióloga

Especialista en Audiología.

Docente Escuela Colombiana de Rehabilitación

ldottor@ecr.edu.co

Leidy Natalia Alvarez Santos

Fonoaudióloga

Estudiante de Especialización en Audiología ECR

nataly.alvarez27@hotmail.com

Jennifer Carolina Rivera Tello

Fonoaudióloga

Estudiante de Especialización en Audiología ECR

jcarolina_1tello@hotmail.com

Yuly Marcela Rodriguez Jimenez

Fonoaudióloga

Estudiante de Especialización en Audiología ECR

yuly_marcela@hotmail.com 


\title{
APLICACIÓN DEL INVENTARIO DE DISCAPACIDAD DEL TINNITUS EN USUARIOS DE IPS EN BUCARAMANGA
}

\section{Application tinnitus hándicap inventory on patients of IPS from Bucaramanga}

Fecha de recepción: 2 de noviembre de 2014 - Fecha de aprobación: 1 de diciembre de 2014

\begin{abstract}
RESUMEN
Las personas que refieren tinnitus describen otros problemas asociados que requieren de atención y de medición para establecer el impacto en la vida diaria. En un porcentaje por encima del $20 \%$ de la población mundial refiere sentir zumbidos en los oídos y alrededor de $102 \%$ manifiesta una alteración significativa. El presente estudio pretende realizar una caracterización de una muestra por conveniencia de usuarios atendidos en una IPS de la ciudad de Bucaramanga mediante el instrumento Tinnitus Handicap Inventory (THI) en su versión adaptada al español que busca que la persona valore la incapacidad del tinnitus de manera subjetiva desde el componente psicoemocional en cada una de las categorías funcional, emocional y catastrófica. Los resultados indican que el 31\% de las personas evaluadas reportan un grado de incapacidad del tinnitus severo y un $26 \%$ entre un grado leve y moderado, a pesar de que un $74 \%$ reporta deficiencia auditiva, existe variabilidad entre los grados de incapacidad y el nivel de audición (Zeman, Koller, Schecklmann, Langguth y Landgrebe, 2012). Este estudio promueve la necesidad de continuar con investigaciones acerca del tinnitus en población colombiana que permitan levantar información epidemiológica que demuestre la necesidad de implementar estrategias de intervención por parte del Fonoaudiólogo Especialista en Audiología dentro del sistema de salud colombiano.
\end{abstract}

PALABRAS CLAVE

Acúfenos, calidad de vida, audición, discapacidad, pérdida auditiva

\section{ABSTRACT}

People refer tinnitus describe other associated problems and requires care to set the measuring impact on daily life. A percentage above $20 \%$ of the world population refers feel ringing in the ears and about 1 or $2 \%$ manifested significant alteration. This study aims to make a characterization of a convenience sample of users treated in an IPS of Bucaramanga by the Tinnitus Handicap Inventory (THI) instrument as adapted to the Spanish version to assess the person's opinion about inability of tinnitus from each of the functional categories catastrophic psycho-emotional component. The results indicate that $31 \%$ of those tested reported a degree of disability of severe tinnitus and $26 \%$ from mild and moderate, although $74 \%$ reported hearing impairment, there is variability between the degree of disability and the hearing level (Zeman, 2012). This study promotes the need for continuing research on the tinnitus in Colombian population to allow lifting epidemiological information demonstrating the need to implement intervention strategies by the Speech therapist audiologist within the Colombian health system.

KEY WORDS

Tinnitus, hearing, handicap, quality of life, disability, hearing loss 


\section{INTRODUCCIÓN}

$\mathrm{E}$ tinnitus al ser un fenómeno acústico que afecta uno de los principales sentidos del cuerpo humano, disminuye en gran medida el nivel de vida de los pacientes, así como, su economía y la convivencia social, ya que aumenta los niveles de estrés en las personas que lo padecen, llegando a presentar irritabilidad y alteración en del ánimo, lo que impide una buena relación y entendimiento con las demás personas de su círculo social (Horderlin, 2011, p.2). Este fenómeno corresponde a ruidos que la persona escucha como si provinieran de uno, ambos oídos o de la cabeza, esto asociado a una percepción negativa puede generar un impacto significativo en las personas en diferentes áreas de su vida. En la población mundial entre el 15 y el $20 \%$ refieren esta dificultad, en población colombiana se hace referencia que entre 8 y 10 millones de personas pueden manifestar tener tinnitus pero sólo para 1 a 2 millones puede llegar a ser un problema significativo (Fagelson, 2015). Por lo cual, desde el ámbito profesional de la Fonoaudiología y de los especialistas en audiología es necesario reportar e investigar acerca de este fenómeno que está siendo más frecuente en los usuarios del país, en donde el alcance del profesional va encaminado a contemplar todo el contexto del individuo con deficiencia auditiva e incluso a establecer los determinantes sociales (Murphy. 2012) que pueden apoyar estos procesos de intervención, en dónde el apoyo de las personas más cercanas influye en los sucesión de cambios en la calidad de vida de usuarios con alguna deficiencia auditiva o comunicativa.

Según Rivas y Ariza (2001) en relación con la descripción clínica afirma:

Los acúfenos o tinnitus también pueden ser sonidos reales o virtuales, los virtuales son el producto de la excitación anormal de cualquiera de las estructuras que integran el aparato auditivo desde el oído externo hasta los centros cerebrales. los acufenos se pueden presentar en procesos patológicos del oído externo tales como tapones de cera otitis externa, en oído medio; como otitis medias agudas o crónicas, obstrucción tubárica, mioclonías de los músculos de los huesecillos, otosclerosis y trastornos de oído interno como la laberintitis, cocleopatías degenerativas, enfermedad de meniére, procesos de SNC, pueden ser focales (tumores anomalías vasculares) o difusos (encefalopatía hipertensiva , esclerosis en placas." (p.61)

Para la evaluación del tinnitus existen diferentes protocolos que buscan establecer las condiciones de salud de las personas que lo refieren; entre los procedimientos establecidos se menciona la audiometría tonal, logoaudiometría, inmitancia acústica, la acufenometría, otoemisiones acústicas y otros procedimientos avanzados que se seleccionan de acuerdo a las particularidades de cada caso (Peña. 2006) y cuyos tratamientos abarcan desde el uso de tecnología como el láser, ayudas auditivas hasta estrategias de consejería para su manejo (Dehkordi. et al 2015).

Sin embargo, es importante incluir aquellos instrumentos o escalas que permiten establecer el impacto en la calidad de vida de estos usuarios, por lo cual, el presente estudio realizó la aplicación del instrumento validado al español por Herraiz (2001) y utilizado también por Peña (2006), conocido como "Tinnitus Handicap Inventory - THI" que categoriza las preguntas en tres sub escalas; nivel funcional que evalúa la incapacidad mental, nivel emocional; que se encarga de medir las respuestas afectivas y la catastrófica que ofrece una idea del nivel de desesperación, si la problemática continua la severidad del acúfeno se verá reflejada en los diferentes aspectos de la vida tales como: estrés emocional y laboral, factores fisiológicos, el desempleo y enfermedades físicas y mentales. Esta importancia está dada porque "el grado de severidad es muy alto debido a que es mayor el número de pacientes con tinnitus que el número de pacientes que buscan tratamiento" (Horderlin, 2011, p.2). Existen otros instrumentos que evalúan diferentes dominios que se encuentran en desarrollo y validación que buscan ampliar la información sobre la calidad de vida y establecer las limitaciones en actividades (Tyler, et al. 2014) pero que aún debido a su proceso todavía no se encuentran validados al español. Por lo cual, el THI adaptado al idioma español aplicado en este estudio ha sido seleccionado para el análisis de la muestra seleccionada.

Así mismo, esta escala establece los intervalos del grado de incapacidad global en donde "no incapacidad" se ubica entre un o y $16 \%$, incapacidad leve entre el 18 y $36 \%$, moderada entre el 38 y $56 \%$ y severa entre el 58 y $100 \%$, esta clasificación permite establecer niveles de impacto del tinnitus en la calidad de vida de los usuarios, lo cual orienta el proceso de intervención junto con la evaluación objetivo de la audición y demás deficiencias relacionadas.

\section{MÉTODO}

Se realiza selección de usuarios por conveniencia de la IPS Audiofon de Bucaramanga en una ventana de tiempo entre el o5 de octubre al 30 de noviembre del año 2014 con el criterio de referir acúfenos o tinnitus al momento de la consulta, posterior a una valoración audiológica básica se invita a participar mediante consentimiento informado y se realizar la aplicación del instrumento de estudio. El presente estudio es de tipo descriptivo cuantitativo debido a que destaca las características o rasgos de una situación, fenómeno u objeto de estudio, ya que se recoge y analizan los datos a través de los conceptos y variables evaluadas mediante el THI. (Fernández. 1995) por lo cual permite detallar cada una de las sub escalas emocional, funcional y catastrófica, permitiendo también cuantificar el grado de incapacidad en relación a valores obtenidos de la evaluación de la incapacidad en pacientes que refieren acúfenos

Los criterios de inclusión y exclusión hacen referencia a usuarios que ingresan a valoración audiológica y que en la entrevista o en el diligenciamiento de la anamnesis inicial manifiesten presentar tinnitus a acufeno como cualquier sonido dentro del oído; estas personas se consideraron candidatos para la aplicación del instrumento independiente de sus niveles de audición previo al consentimiento informado. No fueron aceptados menores de edad dentro del estudio. El instrumento consta de 25 preguntas donde el usuario responde de acuerdo a cada ítem "sí", "a veces" y "no" asignando para el puntaje global de incapacidad del tinnitus un valor de cuatro, dos y cero respectivamente. 
Posterior a la calificación se realiza el análisis descriptivo y relación con los resultados obtenidos en la audiometría tonal, teniendo en cuenta que los usuarios ingresan al servicio para que sea aplicado el procedimiento de sensibilidad auditiva. La descripción de los resultados se muestran de acuerdo a las categorías que evalúa el instrumento y a al porcentaje global del mismo.

\section{RESULTADOS}

El número total de personas evaluadas entre octubre y noviembre del año 2014 fue de 46 usuarios, a quienes se les realizó valoración audiológica mediante audiometría y se registraron datos de identificación, grado y tipo de pérdida, por último la aplicación del instrumento para determinar el porcentaje de incapacidad del tinnitus y las categorías más afectadas, los resultados obtenidos fueron los siguientes:

Del total de personas evaluadas, el $54 \%$ de la población es de género femenino y el $46 \%$ masculino. Los rangos de edad se encuentran entre 19 y 40 años $(24 \%$ ), entre 41 y 60 años $(41 \%)$ y entre 61 y 81 años $(35 \%)$, lo que indica que la población que más refiere sensación de tinnitus en los oídos se ubica por encima de los 41 años. La localización del tinnitus se indaga de acuerdo a si sólo lo percibe en un oído o en ambos, encontrando que el 56.5\% refiere la sensación en uno de los dos oídos, y el 43.5 en ambos. No hay un resultado significativo entre ambos oídos con un 50\% para derecho e izquierdo.

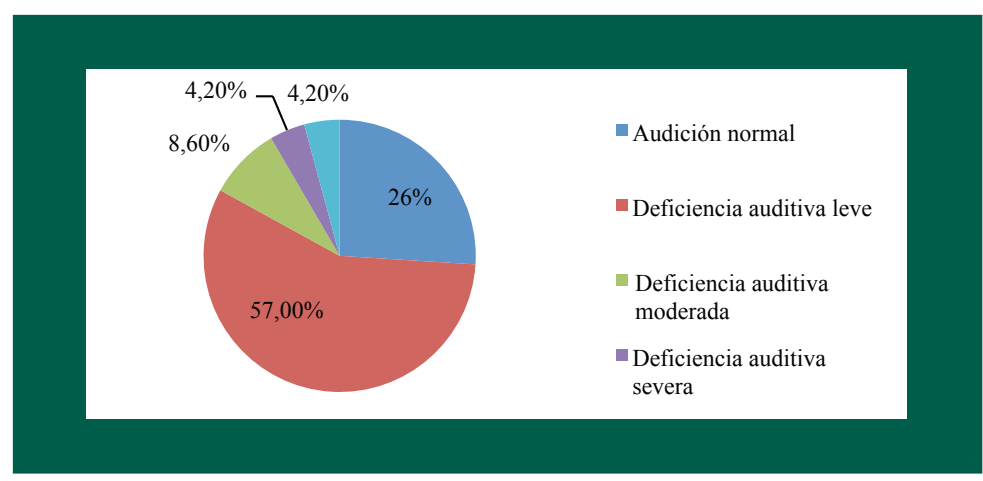

Figura 1. Sensibilidad auditiva de las personas evaluadas.

En la figura 1 se muestra del total de la población evaluada el $74 \%$ de los usuarios presenta algún grado de deficiencia auditiva y un $26 \%$ refiere audición normal, siendo el grado leve el de mayor frecuencia. En la descripción del tinnitus la mayoría manifiesta una sensación de zumbido en un $76 \%$ y entre una chicharra y sonido de turbina el $24 \%$

Los resultados proporcionados después de la aplicación del instrumento sobre discapacidad del tinnitus en cada una de las categorías funcional, emocional y catastrófica evidencia

El primer análisis realizado con respecto al instrumento de estudio fue determinar el grado de discapacidad por categoría, en donde la funcional muestra un mayor impacto con un grado leve del $49 \%$ y moderado del $16 \%$ como se evidencia en la figura 2 , la cual hace referencia a las dificultades para concentrarse o leer, sociolaboral y físi- co. En segundo lugar la categoría emocional que mide las respuestas afectivas como la frustración, enojo, ansiedad y depresión con un $28 \%$ y por último la catastrófica que ofrece una idea del nivel de desesperación y de incapacidad para solucionar el problema referido por el paciente (Herraiz, Hernández, Plaza y Tapia, 2001). Lo cual indica que de la población evaluada que refieren tinnitus, presentan dificultades para concentrarse o leer y en un menor porcentaje genera desesperación y problemas para manejar ésta condición, lo cual, a pesar de ser un menor porcentaje, son usuarios que requieren también de atención inmediata.

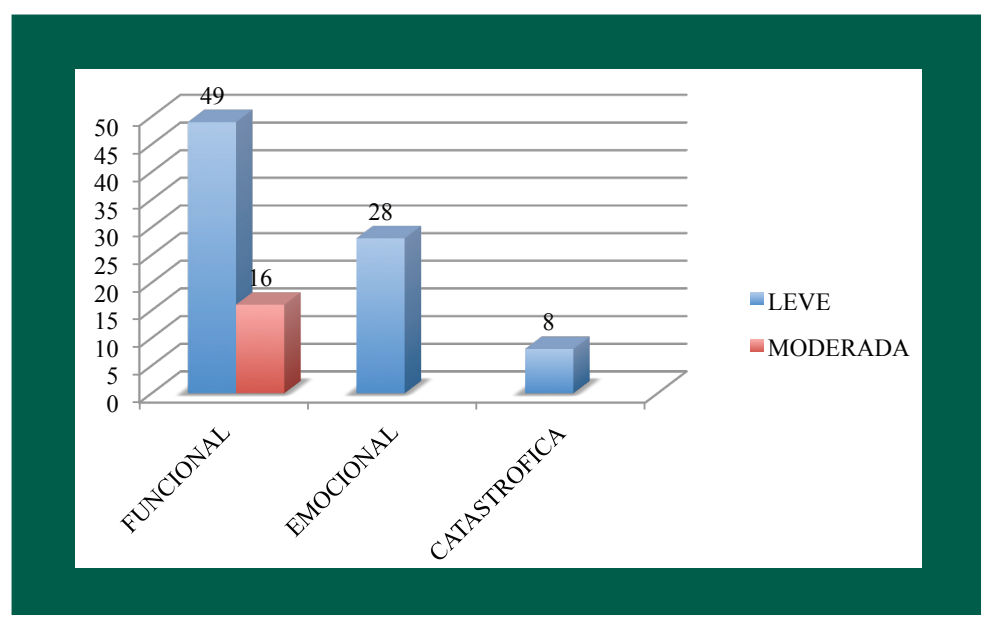

Figura 2. Grados de discapacidad por categoría

En el grado de incapacidad global registrado en el instrumento de estudio evidencia un $31 \%$ de las personas evaluadas tienen un impacto severo en la calidad de vida ocasionada por el tinnitus y entre leve y moderado un $26 \%$ para cada caso con un $17 \%$ cuyo resultado no se relaciona con incapacidad generada por el tinnitus (Figura 3 ).

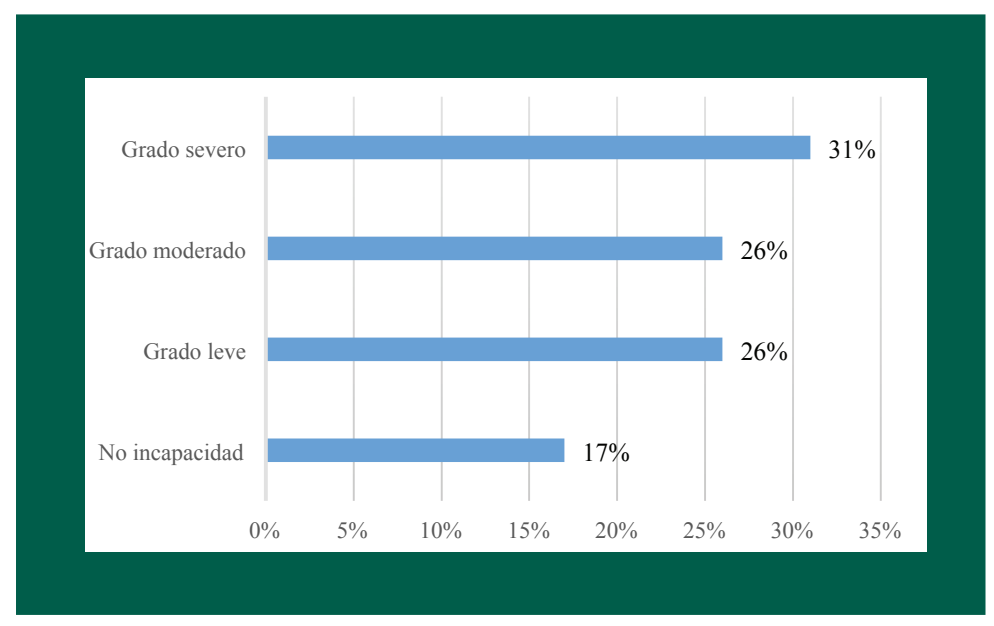

Figura 3. Grado global de incapacidad del tinnitus

La figura 4 hace referencia el rango de respuestas de la escala del instrumento. El comportamiento obtenido en cada uno de las 25 ítems del instrumento, al cual se asigna una calificación de acuerdo a la respuesta cualitativa del usuario evaluado, en donde se eviden- 
cia una mayor respuesta entre el rango o y 2 siendo o como nunca "no" presenta problemas, 2 "a veces" y 4 "siempre"

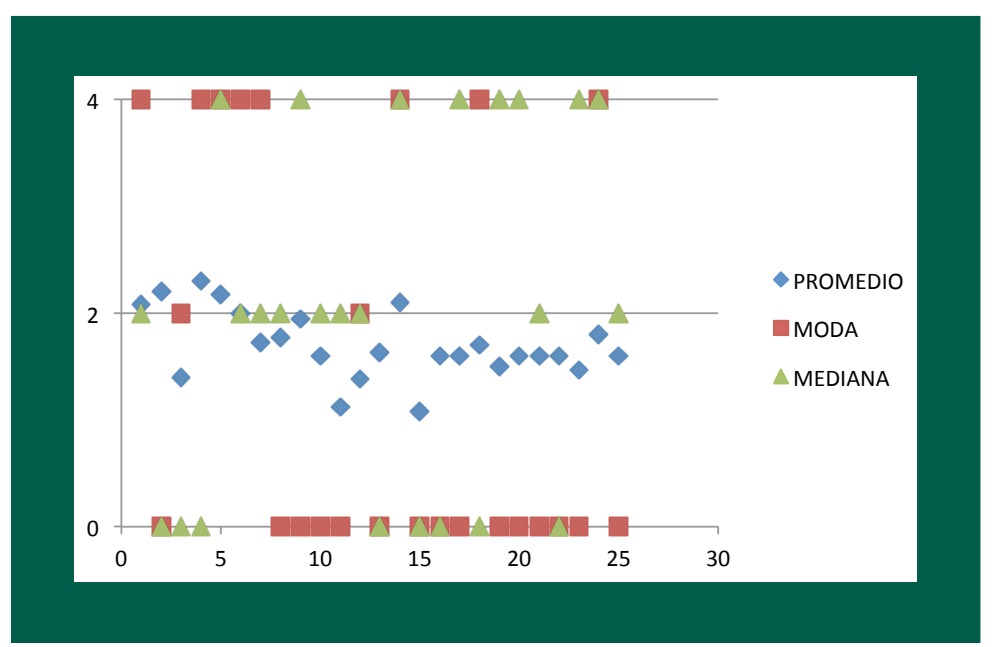

Figura 4. Promedios de respuesta del instrumento en estudio

De las personas que no desarrollaron incapacidad según el instrumento de estudio, presentan tanto audición normal como pérdida auditiva sensorial de grado de severidad variable. En el grado de severidad leve en relación con el nivel de audición, se observa $41 \%$ con audición normal y un 59\% con hipoacusia sensorial de grado leve. En el grado moderado el $75 \%$ presentan hipoacusia neurosensorial de severidad variable con un $25 \%$ de personas con audición normal y en el grado de incapacidad severo el 85\% presentan hipoacusia de severidad variable con un $15 \%$ de audición normal

\section{DISCUSIÓN}

Estos resultados muestran que, en todos los grados de incapacidad del tinnitus, puede presentarse con niveles de audición dentro de límites normales y en los casos donde se asocia a alguna deficiencia, la mayoría son hipoacusias neurosensoriales, los grados de la pérdida varían en todos sus rangos, sumado al hecho que la categoría más relevante es la funcional lo que genera dificultades para concentrarse o leer. En el caso de los porcentajes que indican no incapacidad, no se observa una relación directa con el estado auditivo, ya que la mitad de la población tiene audición normal y la otra mitad presenta pérdida auditiva sensorial leve. Por lo cual, que el estado auditivo pese a ser reportado en la mayoría de la población, la severidad o grado de incapacidad del tinnitus no depende necesariamente de los niveles de audición sino que muestra otros factores afectados por el tinnitus los cuales evalúa el instrumento de estudio. Lo anterior evidencia la importancia y el interés en el abordaje de personas con tinnitus desde el campo de acción de los Fonoaudiólogos Especialistas en Audiología en Colombia ya que esta condición genera impacto en la calidad de vida del usuario influenciado por su estado auditivo y que también influencia la comunicación en relación con el manejo de relaciones y la sensación de inseguridad de quienes refieren tinnitus.

El estudio del tinnitus en población colombiana permite levantar datos epidemiológicos que demuestren la necesidad de implementar estrategias de intervención de estos usuarios. La valoración audiológica básica no es suficiente para establecer u orientar el manejo de esta condición, para ello es necesario adaptar instrumentos subjetivos que muestre la percepción de la persona frente al tinnitus como el aplicado en este estudio o incluso instrumentos que puedan medir otros dominios relacionados con el tinnitus que aporten nueva información para entender e intervenir adecuadamente, que puede verse reflejado en limitaciones para actividades de la vida diaria o restricciones en la participación en situación vitales. En la medida que se tenga en cuenta la importancia a este fenómeno acústico, se podrá intervenir de manera efectiva para solucionar este problema.

El presente estudio se centra sólo en mostrar los resultados en las diferentes categorías analizadas en el instrumento, no se realiza una evaluación formal del tinnitus, por lo cual, se sugiere en posteriores investigaciones realizar una valoración exhaustiva que pueda clasificar el mismo, y genere orientaciones para la intervención de acuerdo con el tipo, nivel o clasificación encontrada. Este tipo de estudios se han realizado en otros países, por lo que es necesario establecer este comportamiento en la población colombiana para que este tipo de tratamiento o abordaje pueda incluirse dentro del sistema de salud del país, que incluya no sólo la deficiencia, ya que esta condición no sólo tiene consecuencias en la persona sino que afecta todo su entorno, por lo cual también puede ser de interés establecer el papel de la familia o el apoyo social cuando se proyecte la intervención de personas con tinnitus. 
